\title{
International Neonatal Consortium Terminology
}

National Cancer Institute

\section{Source}

National Cancer Institute. International Neonatal Consortium Terminology. NCI

Thesaurus. Code C155946.

Terminology developed by the International Neonatal Consortium (INC), a C-Path consortium formed to forge a predictable regulatory path for evaluating the safety and effectiveness of therapies for neonates. Operating in the pre-competitive space, INC addresses the need for measurement and assessment of clinical outcomes in neonates through teams that share data and expertise to advance regulatory science. 